\title{
A neutral cue facilitates detection of a visual target by modulating attention
}

\author{
CARLOS M. HAMAME, PAUL H. DELANO, and LUIS ROBLES
}

Physiology and Biophysical Program, Institute of Biomedical Sciences, Faculty of Medicine, Universidad de Chile, Santiago, Chile.

\begin{abstract}
Twelve rats were trained to perform a two-choice visual detection task in which a right or left light was presented and the animals were required to press the lever located under the illuminated light for a food reward. In seventy percent of the trials the target light was preceded by presentation of a neutral cue (a central light). Relevance of the neutral cue for detection of the target was analyzed by comparing behavioral indices of attention in its presence and absence. Accuracy was significantly higher in presence than in absence of the neutral cue, while mean response latencies were lower in presence than in absence of the neutral cue. These results indicate that the animals allocated attentional resources on the target detection during a high expectancy period after the onset of the neutral cue. This could facilitate target detection and improve the performance in the presence of the neutral cue.
\end{abstract}

Key terms: alerting effect, attention, detection task, expectancy, neutral cue, rat.

\section{INTRODUCTION}

Attention has been described in different terms, as an early sensory filter that allows the most relevant information to be processed by the nervous system, either in terms of a blockade (Broadbent, 1958) or attenuation (Treisman, 1969) of irrelevant information, or as a later filter system that acts during the motor response selection (Deutsch and Deutsch, 1963). An alternative model describes attention as the process of allocation of cognitive resources to different stimuli from the environment depending on their relevance for the organism and on the perceptual load that they represent in a given context (Lavie, 1995). On the other hand, studies that use animal models, mainly rodents, have focused on an approach to attention that compromises several processes, such as vigilance or sustained attention, selective attention, divided attention, orienting (Bushnell, 1998; Muir, 1996), and expectancy (Pang et al., 1992;
Pang et al., 1993). This perspective has encouraged the development of different kinds of behavioral tasks for dissecting attention. The main purpose is to relate rodents' performance in behavioral tasks to the above-mentioned attentional processes, attempting to develop rodent models of human attentional functions (see Robbins, 2000; Bushnell, 1998; Muir, 1996 for reviews on the subject).

Visual two-choice reaction time tasks have been extensively used in the assessment of attentional processes in rodents (Bushnell, 1995; Delano et al., 2007; Grilly and Gowans, 1988; Grilly et al., 1998; Hamame et al., 2006; Sahgal, 1988; Stoher et al., 1997), usually relating attention to some physiological and pharmacological variables. The way in which a particular two-choice task involves a specific attentional process depends on task parameters such as: type, intensity, timing and duration of targets, nature of the reward and inter-trial interval (ITI) duration. 
In sustained-attention studies the critical parameter is the predictability of the target that it is related to, among others factors, the duration and variability of the ITI and the absence of stimuli that could serve as cues for target presentation (e.g.: Grilly and Gowans, 1988; Sahgal, 1988).

Another kind of two-choice task makes use of cues, which are stimuli that are presented before a target and that contain some sort of information about the occurrence of it, to orient attention and modulate stimulus detection. This kind of paradigm avoids the vigilance component of attention and allows the assessment of other processes, such as attentional shift (Stoher, et al., 1997), or selective attention in a delimited period of time (Delano, et al., 2007). Cued-detection paradigms have been extensively used in measurements of human covert orienting and also in attempts to model human covert orienting in rats (Bushnell, 1995). However, since it is not possible to control or monitor visual fixation in rats, these attempts only achieved a cued-detection paradigm, termed overt orienting. In this behavioral procedure, rats are exposed to a visual cue that signals the location in which the target is going to appear (i.e., left or right in the operant chamber) and several conditions are tested, such as: no cue, different timing between cue and target onset, invalid cues and neutral cue (onset of the two cues).

Bushnell's data showed that valid cues (i.e., ones that signal correct target location) improved and invalid cues (i.e., ones that signal an incorrect location) decreased performance of rats, while neutral cues had little effect on accuracy and reaction time compared to the no-cue condition.

In contrast, in a previous work, we found clear behavioral differences between the no-cue and neutral cue (NC) conditions (Hamame, et al., 2006). In that work, we studied the relevance of a NC in a twochoice visual detection task by comparing the behavioral performance of rats during five days in which a $\mathrm{NC}$ was presented and five subsequent days in which the $\mathrm{NC}$ was removed. The crucial finding was that the NC improved performance in the task by increasing expectancy and attentional load during the periods of presentation of visual stimuli. However, since the $\mathrm{NC}$ removal changed the original context of stimuli to which the rats were trained, there was the possibility that the change of performance in the task was due to a generalization decrement.

In the present study, we use a two-choice visual detection task similar to the one used previously (Hamame, et al., 2006). However, in this case only $70 \%$ of the trials started with the presentation of a NC before the onset of the target lights and $30 \%$ of the trials had no NC. This experimental design allowed us to assess the relevance of the NC in performance, avoiding the occurrence of generalization decrement. We are now verifying that the $\mathrm{NC}$ allows the rats to delimit a high expectancy period during the task, in which they are able to allocate cognitive resources to target detection.

\section{METHOD}

\section{Subjects}

Twelve male Long Evans rats (from the animal facility of the Faculty of Medicine of the University of Chile), weighing between 250-350 $\mathrm{g}$ at the start of training, were used. They were individually housed in temperature-controlled conditions, with an alternating dark-light cycle (lights off from 8:00 to 20:00 hrs). All rats were given water ad libitum and were food deprived, maintaining 80 to $90 \%$ of their free-feeding weight during the experimental period. All animals were trained five days a week, over approximately two months with two to four sessions of 50 trials each day, except for the testing phase that consisted of one session of 110 trials on four consecutive days.

\section{Apparatus}

The apparatus consisted of a mesh operant cage $(25 \mathrm{~cm} \times 31 \mathrm{~cm} \times 31 \mathrm{~cm})$ with an aluminum front panel and a metal grid floor. The front panel had three lights: a central light (neutral cue) above a magazine food dispenser and two lateral lights (targets) above two levers that were located 
$6 \mathrm{~cm}$ above the grid floor. The target lights, central light and the magazine food dispenser were $10,14.5$ and $0.5 \mathrm{~cm}$ above the floor, respectively.

\section{Behavioral procedure}

Twelve food-deprived rats were trained to perform a two-choice task that consisted of a visuospatial discrimination. Stimuli presentation and data acquisition were controlled by a National Instruments ${ }^{\circledR}$ board (PCI-6040) housed in a PC (Pentium III), using a custom-made computer program (Labwindows $\mathbb{C}$ system) and a digital interface with a time resolution of 1 ms. After initial shaping of lever pressing for the food reward (dustless precision pellets, $45 \mathrm{mg}$; Bioserv (C) for two to five days, the animals were trained to respond to illumination of the right or left light by pressing the lever under the light to obtain a food pellet. Seventy percent of the trials were randomly selected to begin with the onset of the NC (central light) with a random duration (2-4 s). On the other $30 \%$ of the trials there was no presentation of the NC during the 2-4 s before target presentation. Similar ratios of cue presentation have been used in human studies designed to assess the role of cues in the process of attentional orienting (Posner, 1980, Posner et al., 1980). Animal models of cued orienting have also used this specific ratio of NC presentation to ensure learning, while still having trials without NC (Bushnell, 1995; Chiba et al., 1999; Delano et al., 2007). Immediately after presentation of the $\mathrm{NC}$, the right or left light was presented (pseudo-randomly chosen, with probability 0.5). During training the ITI duration was 7-13 s (randomly chosen) and the target duration was progressively reduced from 10 to $0.5 \mathrm{~s}$ along sessions, maintaining in both conditions, with and without $\mathrm{NC}$, an accuracy $\geq 80 \%$ and percentage of omissions $\leq 20 \%$ (see below for definitions of accuracy and omissions). During training animals were expected to meet these behavioral criteria to start the testing phase, which consisted of one daily session of 110 trials for four consecutive days. During these test sessions several behavioral indicators (specified below) were measured to compare the performance between the trials with and without NC.

\section{Behavioral variables and data analysis}

The specific behavioral variables measured were: latency of correct responses (time between target-light onset and correct leverpressing response), accuracy ([correct responses / (correct responses + incorrect responses)] $x$ 100) and omission percentage ([number of trials without response / total number of trials] $x$ 100). The first 10 of the 110 trials of test sessions were discarded from indices computation to let the animals habituate to the operant chamber.

Values of behavioral indices measured in presence and absence of the NC were compared using a $t$-test for matched pairs. All statistical analyses were performed using the computational program SPSS 13(C). The criterion for significance in all tests was $\alpha=.05$.

\section{RESULTS}

The twelve trained rats accomplished the criteria of accuracy $(>80 \%)$ and the percentage of omissions $(<20 \%)$ before beginning of the testing phase, not only in the average values for all trials, but also when considering separately the trials with and without NC.

In order to find out whether the presence of the NC produces facilitation in target discrimination, through a process of attentional orienting, three behavioral indices (mean latency, accuracy and omissions) were evaluated in the presence and absence of the NC. Mean latency of correct responses was significantly lower in trials with $\mathrm{NC}$ than in trials without $\mathrm{NC}$ (Figure 1A, t $(11)=-2.208, \mathrm{p}<0.05)$. In contrast, accuracy was significantly higher in the presence than in absence of the $\mathrm{NC}$ (Figure 1B, t $(11)=3.764, \mathrm{p}<0.005)$. Finally, no significant difference was found in the percentage of omissions between the two conditions (Figure 1C, t $(11)=-0.959$, $\mathrm{p}=0.358)$. 

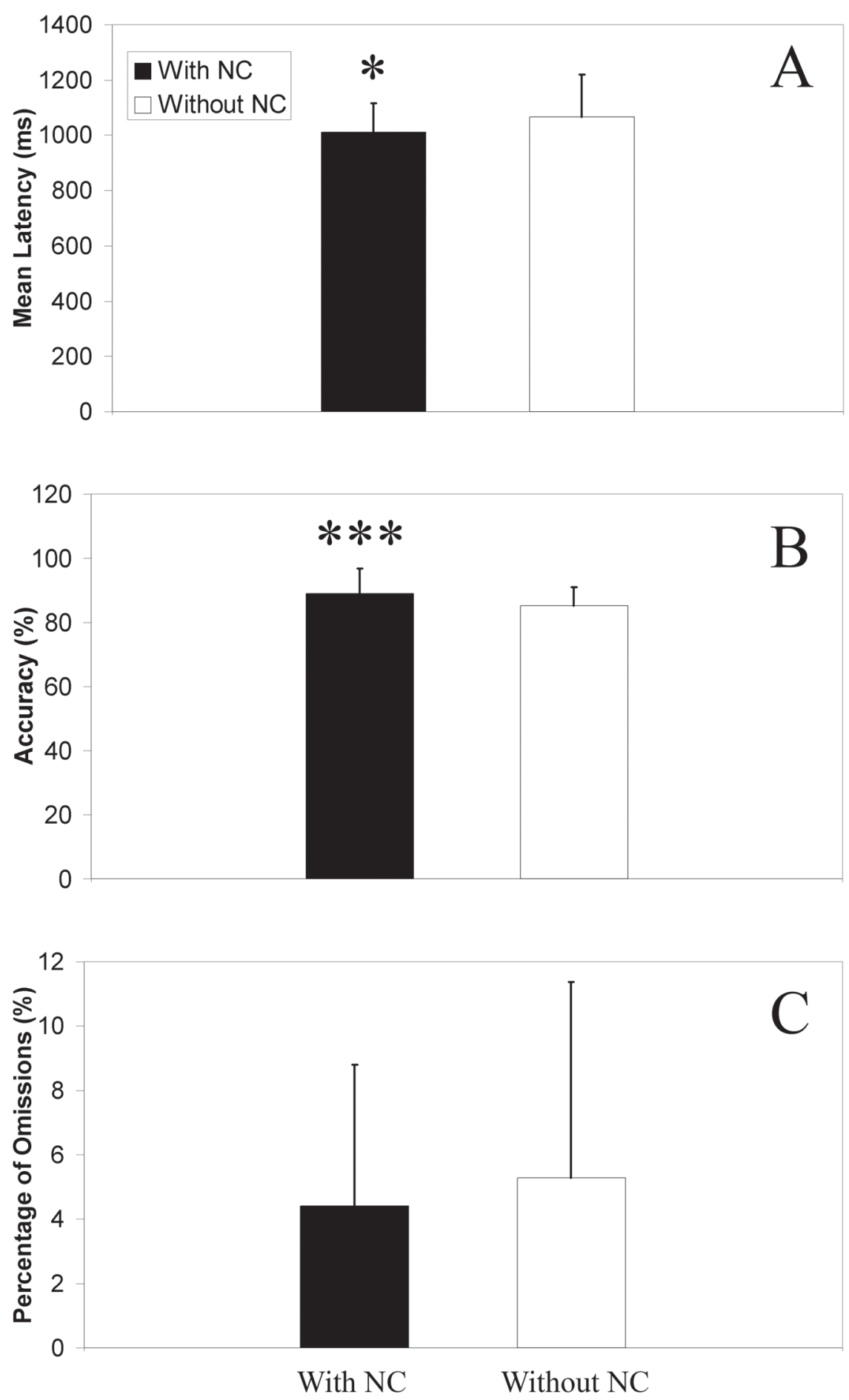

Figure 1: Comparisons of behavioral indices in the presence and absence of the neutral cue A) Mean response latency was $1011 \mathrm{~ms}(S D=119)$ in the trials with $\mathrm{NC}$ and increased to $1066 \mathrm{~ms}(S D$ $=140)$ in the trials without NC. B) Accuracy decreased from $89.04(S D=6.06)$ in the trials with NC to $85.06(S D=7.79)$ in the trials without NC. C) The percentage of omissions increased from $4.4(S D=4.39)$ in presence to $5.28(S D=6.11)$ in absence of the NC, although not reaching significance. Error bars $(S D)$ are depicted, and significance is indicated by: $*=p<0.05$ and $* * * p$ $<0.005$. 


\section{DISCUSSION}

This study evaluated the relevance of a NC on the performance of rats in a two-choice detection task, using behavioral variables that are traditionally associated with attentional processes, such as latency of response, omissions and accuracy. We demonstrated that the presence of a $\mathrm{NC}$ anticipating the presentation of a target light improves the performance in the detection task.

The improvement of performance in the attentional task in the presence of the $\mathrm{NC}$ is probably due to an increase in the animals' expectancy, which allows them to allocate attentional resources on target detection during a limited period of time, in contrast to a more constant demand in absence of the NC.

\section{Latency of response}

Latency of response, also called reaction time, has been extensively used as a measure of attention in detection tasks. In this case, mean response latencies of correct responses were lower in presence than in absence of the NC. In a previous study, Bushnell (1995) used a cued target detection task, in which cues could be valid (signaling correct target positions), invalid (signaling incorrect target positions) and neutral (signaling timing but no target position). Bushnell (1995) found little effect of the NC over the no-cue condition in accuracy and latency of the responses. Those results contrast with our findings, in which the presence of a NC significantly decreased the mean latency. The fact that we also found significant differences in accuracy between the cue and no-cue conditions led us to conclude that the different results obtained in both studies could be explained by differences in the properties of the NC used. In Bushnell's task the NC consisted of the simultaneous presentation of two valid cues, in contrast to our use of a central light as a NC, without relation to any other cue. In Bushnell's experiments there is still a possibility of an association between each of the cues and the side in which the target may appear; in this sense our NC was more neutral. There were also other differences in experimental design that could explain the differences between Bushnell's and our results, such as his use of a smaller ITI (5s) and different stimulus onset asynchronies between NC and target.

In a recent study (Hamame et al., 2006) we showed that the presence of a NC, similar to the present one, produced response latency reductions in a two-choice detection task, which were larger than in the present work. However, in that study, rats were trained in the presence of the NC, which was removed after the rats performed at a certain behavioral criterion for five consecutive days. The main problem with that experimental design is that when an animal has learned to respond in a certain stimuli arrangement, any variation on that original training context may produce a change in the animal's response. Consequently, a generalization decrement (see Ghirlanda and Enquist, 2003, for a review on the subject) could partly explain the differences in latency and other behavioral indices reported by Hamame et al. (2006). In the present work, in which we also found latency differences between the trials with and without NC, although lower than in the previous study, animals were trained from the beginning in a task in which the NC was presented in only $70 \%$ of the trials, thus, a generalization decrement effect can be discarded.

In summary, we think that the alerting effect of the NC facilitates, through temporal and spatial allocation of attentional resources, the setting of a readier perceptual system, so that target detection and response emission occur faster in the trials with NC presentation.

\section{Omissions}

Percentages of omissions were also compared in the presence and absence of the NC, under the assumption that a more attentive animal would make less omission errors than an inattentive one. However, there was no significant difference in the percentage of omissions between the trials with and without NC. If we consider that 
after each target detection there is a release of attentional resources, it is possible that a 10 -seconds ITI was not enough to see an effect of the $\mathrm{NC}$ on omission errors.

\section{Accuracy}

Accuracy, as a measure of the rat's capability to discriminate between stimuli, is an important index for correct detection and identification of the target. As expected, accuracy was higher for trials with NC presentation than without. The presence of a NC that turns the target presentation into a predictable event in time makes sustained attention unlikely. However, in the $30 \%$ of the trials without $\mathrm{NC}$, target detection was more difficult, since its occurrence was less predictable and the rat had to be more attentive during the entire ITI period for good performance.

In addition to this behavioral evidence on the relevance of the $\mathrm{NC}$ for modulating rats' attention, in physiological experiments we have demonstrated that the presence of a $\mathrm{NC}$ in a selective attention task reduces auditory sensitivity in chinchillas attending to visual targets (Delano, et al., 2007). Together, these results show the convergence of behavioral and physiological evidence on the relevance of the NC in modulating attention during a delimited period of time.

\section{GENERAL CONCLUSIONS}

Taken together, these data on mean latencies, percentage of omissions and accuracy constitute an extensive evaluation of the role of a $\mathrm{NC}$ in modulating attention in a two-choice task. The results clearly demonstrate that a NC improves performance of rats in a two-choice detection task. The most probable explanation for this improvement is that the NC facilitates target detection, first by turning the target into a predictable event in time, thus allowing the animals to make a better distribution of attentional resources during the session, and second, by augmenting expectancy just before target presentation, thus getting the animals in a readier state for detection and identification of targets.

\section{ACKNOWLEDGEMENTS}

We dedicate this publication to Professor Teresa Pinto-Hamuy who encouraged our work in this research project with great enthusiasm. This work was supported by FONDECYT 1020970 and 1080227 , CONICYT to CMH and Fundación Puelma, PG-42-2004 to PHD. Special thanks to Fernando Vergara for technical assistance.

\section{REFERENCES}

BROADBENT D (1958) Perception and communication London: Pergamon Press

BUSHNELL PJ (1995) Overt orienting in the rat: parametric studies of cued detection of visual targets. Behav Neurosci 109: 1095-1105

BUSHNELL PJ (1998) Behavioral approaches to the assessment of attention in animals. Psychopharmacol 138: 231-259

CHIBA AA, BUSHNELL PJ, OSHIRO WM, GALLAGHER M (1999). Selective removal of cholinergic neurons in the basal forebrain alters cued target detection. Neuroreport 10: 3119-3123

DELANO PH, ELGUEDA D, HAMAME CM, ROBLES L (2007) Selective attention to visual stimuli reduces cochlear sensitivity in Chinchillas. J Neurosci 27: 4146-4153

DEUTSCH J, DEUTSCH D (1963) Attention: Some theoretical considerations. Psychol Rev, 70: 80-90

GHIRLANDA S, ENQUIST M (2003) A century of generalization. Anim Behav 66: 15-36

GRILLY DM, GOWANS GC (1988) Effects of naltrexone, and $d$-amphetamine, and their interaction on the stimulus control of choice behavior of rats. Psychopharmacol 96: 73-80

GRILLY DM, PISTELL PJ, SIMON BB (1998) Facilitation of stimulus detection performance of rats with $d$ amphetamine: a function of dose and level of training. Psychopharmacol 140: 272-278

HAMAME CM, DELANO PH, ROBLES L (2006) Relevance of a neutral cue in a two-choice task. Biol Res 39: 259-267

LAVIE N (1995) Perceptual load as a necessary condition for selective attention. Journal of Experimental Psychology: Human Perception and Performance 21: 451-468

MUIR JL (1996) Attention and stimulus processing in the rat. Cog Brain Res 3: 215-225

PANG K, MERKEL F, OLTON DS (1992) Expectancy and stimulus frequency: a comparative analysis in rats and humans. Percept Psychophys 51: 607-615.

PANG K, WILLIAMS MJ, EGETH H, OLTON DS (1993) Nucleus basalis magnocellularis and attention: Effects of muscimol infusions. Behav Neurosci 107: 10311038

POSNER MI (1980) Orienting of attention. Q J of Exp Psychol 32: 3-25 
POSNER MI, SNYDER CRR, DAVIDSON BJ (1980). Attention and the detection of signals. J Exp Psychol 109: $170-174$

ROBBINS TW (2000) Arousal and attention: psychopharmacological and neuropsychological studies in experimental animals. In: Parasuraman R (ed), The attentive brain. London: The MIT Press. pp. 189-220

SAHGAL A (1988) Vasopressin and amphetamine, but not desglycinamide vasopressin, impair positively reinforced visual attention performance in rats. Behav Brain Res 29: 35-42

STOHER JD, MOBLEY SL, ROICE D, BROOKS R, BAKER L, WILEY RG, WENK GL (1997) The effects of selective cholinergic basal forebrain lesions and aging upon expectancy in the rat. Behav Neurosci 76: 214-227

TREISMAN AM (1969) Strategies and models of selective attention. Psychol Rev 76: 282-299 